\title{
Métodos não-invasivos para análises hormonais aplicadas aos estudos de ecologia e etologia
}

\author{
Ricardo José Garcia Pereira'
}

\begin{abstract}
1 - Doutorando pelo Departamento de Reproducao Animal. FCAV/UNESP. Docente no curso de Medicina Veterinaria da Universidade de Franca. E.mail: ric jose_garcia@hotmail.com
\end{abstract}

RESUMO - O monitoramento endócrino pela mensuração de metabólitos urinários e fecais de hormônios esteróides tem se mostrado uma alternativa viável na investigação da fisiologia reprodutiva e do estresse em uma grande variedade de aves e mamíferos, domésticos e selvagens. Esta abordagem tem contribuído para uma maior integração da endocrinologia com estudos comportamentais e ecológicos, gerando informações mais detalhadas em diversas áreas tais como bem-estar animal, comportamento social, reprodução, biologia da conservação, biomedicina, entre outros. Todavia, o emprego desta metodologia no Brasil tem sido limitado principalmente à pesquisa de parâmetros reprodutivos e de estresse em espécies selvagens, não havendo até o momento trabalhos publicados utilizando a quantificação urinária ou fecal de glucocorticóides ou esteróides sexuais em animais domésticos. Desta forma, esta palestra tem como objetivo ilustrar alguns exemplos de estudos conduzidos no país envolvendo técnicas de monitoramento endócrino não-invasivo, assim como expor possíveis áreas de aplicação desta ferramenta em pesquisas com espécies domesticas.

Palavras-chave: Monitoramento não-invasivo, esteróides, endocrinologia, estresse, fezes, reprodução,

ABSTRACT - The endocrine monitoring through urinary and fecal steroid analysis has been proven a feasible alternative in the investigation of reproductive and stress physiology in a great variety of birds and mammals (domestic and wild species). This approach has contributed to enhance integration between endocrinology and behavioral/ ecological studies originating detailed information towards different areas, such as animal welfare, social behavior, reproduction, conservation biology, biomedicine, among others. In Brazil, however, its application has been limited mainly to the determination of reproductive and stress parameters in wild species, with no published articles employing measurement of urinary or fecal glucocorticoids or sexual steroids in domestic animals. Thus, the aim of this lecture is first show examples of Brazilian researches involving non-invasive endocrine monitoring, and secondly illustrate possible applications of this technology in studies involving domestic animals.

Key Words: endocrinology, feces, non-invasive monitoring, reproduction, steroids, stress

\section{Introdução}

Nos últimos 20 anos muitos avanços foram obtidos no aperfeiçoamento de métodos de quantificação de metabólicos urinários e fecais de esteróides, a ponto dos mesmos serem correntemente empregados na investigação das relações endócrino-comportamentais em inúmeras espécies domésticas e selvagens (para maiores informações, ler Peter et al., 1996, Schwarzenberger et al., 1996, Wasser et al., 2000, Palme et al., 2005). No entanto, apesar das facilidades que estas técnicas propiciam na obtenção de amostras seriadas diversos fatores devem ser cuidadosamente consiCorrespondências devem ser enviadas para: ric_jose_garcia@hotmail.com derados durante o seu uso, para que resultados confiáveis e conclusões apropriadas possam ser alcançados (Palme 2005, Wasser et al., 2002). O metabolismo e excreção dos hormônios esteróides variam substancialmente entre espécies, e por essa razão todo monitoramento endócrino não-invasivo deve ser previamente validado para cada espécie antes de sua aplicação (Peter et al., 1996, Schwarzenberger et al., 1996).

Por sua vez, alguns dos testes de validação requerem uma infra-estrutura laboratorial nem sempre disponível em muitas instituições brasileiras, fato que torna o acesso a essa tecnologia restrito a poucos centros de pesquisa. Por exemplo, 
estudos envolvendo a administração de esteróides radio-marcados (necessários tanto para a determinação da rota e tempo de excreção, quanto para o conhecimento dos metabólicos urinários e fecais) exigem o credenciamento do laboratório junto aos órgãos governamentais para que se possam manipular materiais radioativos (p.e. CENAESALQ). Do mesmo modo, poucos grupos de pesquisa no país possuem uma rotina laboratorial para análise de esteróides por cromatografia líquida de alta pressão ou cromatografia gasosa, técnicas igualmente indispensáveis na determinação de metabólicos hormonais.

Por outro lado, aprimoramentos contínuos relacionados à estocagem de amostras, extração de esteróides e imunoensaios vem popularizando o uso desta ferramenta no Brasil, por reduzir significativamente o tempo de processamento do material e os custos referentes à equipagem do laboratório. Como prova disto, estudos recentes demonstraram que o simples congelamento de amostras fecais em freezer convencional $\left(-20^{\circ} \mathrm{C}\right)$ poucas horas após a sua defecação é uma das melhores opções para preservação dos níveis hormonais por períodos prolongados (Millspaugh \& Washburn, 2004; Palme, 2005; Wasser et al., 2002). Concomitantemente, os protocolos de extração de esteróides fecais vem se tornando cada vez mais simplificados (p.e. etapa única de agitação da amostra em metanol:água), resultando na redução de mão-de-obra e diminuição das variações relacionadas a etapas adicionais de extração (Mostl \& Palme, 2002; Palme, 2005). Contudo, o elemento mais expressivo na difusão do monitoramento não-invasivo pelo país é sem dúvida alguma a padronização de sistemas imunoenzimáticos (EIA) em substituição aos radioimunoensaios (RIA). Métodos imunoenzimáticos para quantificação de esteróides apresentam reagentes estáveis, são fáceis de executar (necessitando apenas de equipamentos básicos), exibem uma sensibilidade comparável aos radioimuno-ensaios, e evitam os problemas associados ao uso de materiais radioativos (Hodges 1996, Lee et al., 1995).

Ainda assim, outro obstáculo significativo à realização de trabalhos envolvendo dosagens hormonais é o custo da análise por amostra principalmente quando referimo-nos a utilização de kits comerciais (p.e. em 2006 o custo estimado era de $\mathrm{R} \$ 18$ a 25/ amostra para kits imunoenzimáticos).
Até o momento, grande parte dos estudos hormonais fecais desenvolvidos em universidades brasileiras empregou kits comerciais importados (Chelini et al., 2005; Chelini et al., 2006; Guimarães et al., 2002; Korndorfer 1992; Meirelles, 1993; Morais et al., 1996; Morais et al., 2002; Morato et al., 2004; Muhlbauer et al., 2006; Pereira et al., 2005; Pereira et al., 2006a; Pereira et al., 2006b; Pinho, 2004; Ribeiro, 1994). Neste contexto, uma alternativa adotada por algumas instituições nacionais é a compra de reagentes separados e subseqüente montagem dos imuno-ensaios, saída que vem proporcionando uma redução substancial nos gastos laboratoriais. Porém, permanecem as dificuldades relacionadas à importação de alguns reagentes imunoenzimáticos, especialmente de anticorpos e conjugados, fato que pode gerar atrasos consideráveis na implantação destas opções.

Como pode ser observado na Tabela 1, diversas espécies de mamíferos e aves (domesticas e selvagens) já foram pesquisadas no Brasil e no exterior por meio de técnicas endócrinas nãoinvasivas no Brasil e no exterior. Entretanto, um aspecto curioso que também pode ser notado nesta tabela é a enorme carência no país de trabalhos hormonais fecais envolvendo animais domésticos. Esta lacuna está provavelmente correlacionada:

1) a falta de conhecimento dos pesquisadores brasileiros sobre o emprego desses métodos,

2) a habituação em se realizar estudos endócrinos a partir de amostras sanguíneas, ou

3) aos problemas de exeqüibilidade anteriormente mencionados. Portanto, o intuito desta palestra é exemplificar alguns resultados obtidos em projetos feitos no país (já desenvolvidos ou em fase de execução), além de listar algumas possibilidades de aplicação de tais abordagens em estudos ecológicos e/ou ecológicos com animais domésticos.

\section{Exemplos da utilização de glucocorticóides fecais}

Todos os trabalhos empregando glucocorticóides fecais desenvolvidos no Brasil até hoje objetivaram mensurar o estresse ou bem-estar animal de espécies selvagens frente a condições

๑ 2007 Sociedade Brasileira de Zootecnia 
Tabela 1 - Grupos de aves e mamíferos já estudados com o auxílio de técnicas de monitoramento endócrino não-invasivo no Brasil e exterior.

\begin{tabular}{l|l|l|l}
\hline Localidade & Classificação & & Grupos animais \\
\hline \multirow{2}{*}{ Exterior } & Domésticos & $\begin{array}{l}\text { Aves } \\
\text { Mamíferos }\end{array}$ & $\begin{array}{l}\text { Galinhas, codornas e gansos } \\
\text { Gatos, cães, porcos, ovelhas, bois e cavalos }\end{array}$ \\
\cline { 3 - 4 } & Selvagens & Aves & $\begin{array}{l}\text { Beija-flores, calaus, cormorões, grous, mergulhões, } \\
\text { passarinhos, pingüins, pombos, papagaios,calopsistas } \\
\text { periqueitos, águias, açores, falcões, faisóes e perdizes. }\end{array}$ \\
\cline { 3 - 4 } & Mamíferos & $\begin{array}{l}\text { Brisões, abras selvagens, lobos, raposas, hienas, cães } \\
\text { selvagens, cervos, veados, baleias, tamanduás, } \\
\text { elefantes, tigres, leões, leopardos, linces, lebres, } \\
\text { gambás, morcegos, furões, chimpanzés, gorlias, } \\
\text { sagüis, micos, rinocerontes, camundongos e ursos. }\end{array}$ \\
\hline \multirow{2}{*}{ Brasil } & Domésticos & Aves & - \\
\cline { 3 - 4 } & Mamíferos & Coelhos. \\
\cline { 2 - 4 } & Selvagens & Aves & Papaguaios e falcões. \\
\cline { 3 - 4 } & Mamíferos & $\begin{array}{l}\text { Cervos, veados, peixes-boi, bichos-preguiça, gatos- } \\
\text { do-mato, jaguatiricas, onças, jaguarundis, macacos, e } \\
\text { hamsters. }\end{array}$ \\
\hline & & & \\
\hline
\end{tabular}

de cativeiro e/ou vida livre. Um exemplo da aplicabilidade destes compostos na determinação do estresse sob circunstâncias naturais foi o estudo feito por Pereira et al. (2006), no qual diferentes fatores ambientais (p.e. estação do ano, ciclo de chifres, presença humana, reprodução e agrupamento) foram avaliados em relação ao estresse de machos de veado-campeiro (Ozotoceros bezoarticus) de vida livre (Figura 1). Já em cativeiro, Moreira (2001) observou que elementos como tamanho reduzido e baixa qualidade dos recintos (p.e. sem esconderijos, vegetação, puleiros, etc) tinham uma correlação positiva com os níveis fecais de glucocorticóides em fêmeas de gato-do-mato-pequeno (Leopardus tigrinus) e gato-maracajá (Leopardus wiedii) (Figura 2) (Moreira 2001).

Pela quantificação de glucocorticóides fecais, Morato et al. (2004) também pôde comprovar a resposta adrenocortical de machos de onça-pintada (Panthera onca) em relação aos procedimentos de contenção química e eletroejaculação Similarmente, a análise conjunta de parâmetros cardiorespiratórios e glucocorticóides fecais possibilitou avaliar os efeitos neurolépticos da pipotiazina em veados-catingueiros mantidos em cativeiro (Pinho, 2004). Além disso, Chelini et al. (2006) relatou o uso dos metabólicos fecais de cortisol no acompanhamento do impacto de condutas cirúrgicas (p.e. ovarioectomia) em fêmeas de hamster (Mesocricetus auratus). Em resumo, esses exem-
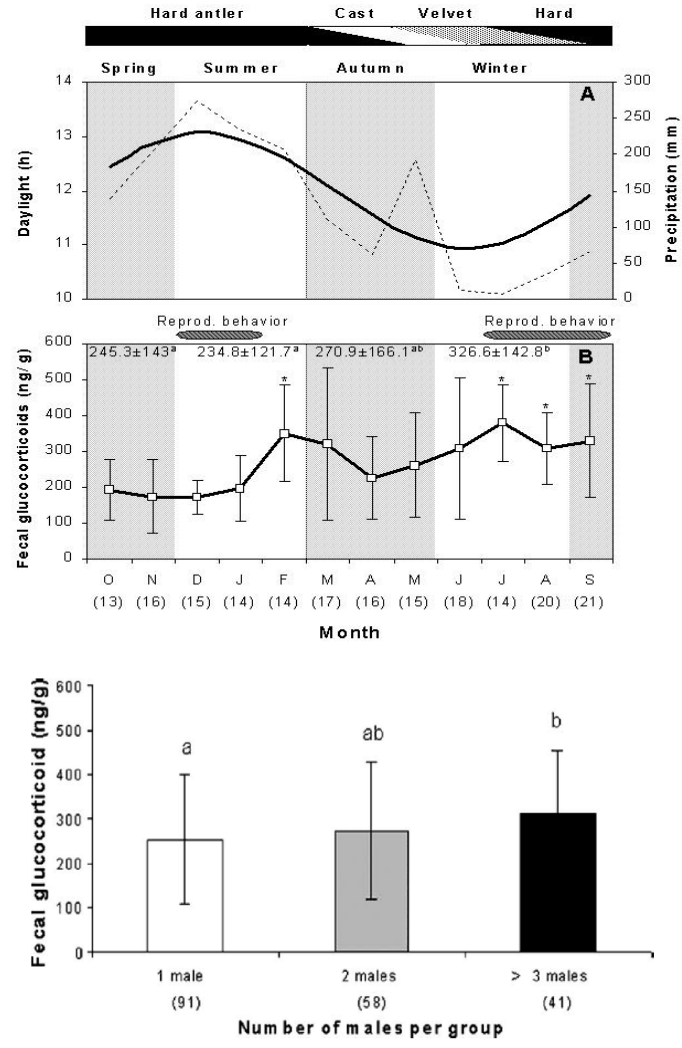

Figura 1 - Resultados de glucocorticóides fecais em relação a diferentes fatores ambientais (p.e. estação do ano, ciclo de chifres, reprodução e agrupamento) em machos de veado-campeiro (Ozotoceros bezoarticus) de vida livre (fonte: Pereira et al., 2006a).

plos apenas reforçam a flexibilidade que a dosagem de esteróides fecais concede a investigação de processos endócrino-comportamentais,

๑ 2007 Sociedade Brasileira de Zootecnia 


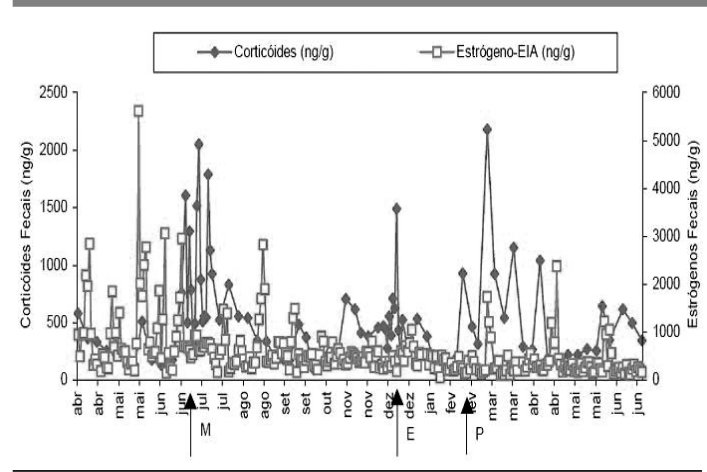

Figura 2 - Perfis de excreção fecal de estrógenos (quadrados vazios) e corticóides (losangos preenchidos) em fêmeas adulta de gato-do-mato-pequeno (Leopardus tigrinus). $\mathrm{M}$ - mudança para recinto pequeno e estéril/ E enriquecimento ambiental do recinto/ $\mathrm{P}$ - perda de pelo (fonte: Moreira 2001).

uma vez que dificuldades como tamanho e comportamento do animal, número de amostras sanguíneas necessárias ou influência da manipulação excessiva nos níveis plasmáticos de cortisol, podem ser superadas sem prejuízos na qualidade do experimento.

Entre as perspectivas científicas que a dosagem de glucocorticóides fecais pode originar no estudo dos animais domésticos estão: comparação de diferentes sistemas de manejo no bem-estar animal (p.e. graus de adensamento em galpões de frangos de corte, estresse térmico induzido por diferentes instalações para suínos, meios de transporte em bovinos, provas de enduro em eqüinos de esporte, etc), monitoramento de tratamentos e evolução de casos clínicos (p.e. cólica em eqüinos), avaliação do comportamento social de diferentes raças, entre outras.

\section{Dosagem de metabólicos fecais de esteróides sexuais}

Aplicações reprodutivas envolvendo a análise de andrógenos, estrógenos e progestinas urinários e/ou fecais juntamente com observações comportamentais são abundantes quando falamos de trabalhos realizados por grupos brasileiros. Aliás, na maioria dos casos, acompanhamentos etológicos dos animais são sempre desejados para que os resultados endócrinos obtidos possam ser melhor interpretados. Por exemplo, a caracterização de ciclos estrais em espécies selvagens torna-se mais segura quando aliamos essas duas variáveis, como pode ser observado na Figura 3 (Pereira et al., 2006b). Adicionalmente, os produtos metabólicos destes esteróides sexuais podem fornecer dados fundamentais em tópicos como: diagnóstico de gestação, caracterização de ciclos estrais e períodos gestacionais normais, determinação do momento do parto, investigação de anormalidades dos ciclos reprodutivos, detecção de ovulação, eficiência de métodos contraceptivos, sazonalidade reprodutiva, correlações entre reprodução e comportamento social, etc (Guimarães et al., 2003, Peter et al., 1996, Schwarzenberger et al., 1996).

Desta forma, dados endócrino-reprodutivos relativos aos grupos animais de difícil manejo (p.e.
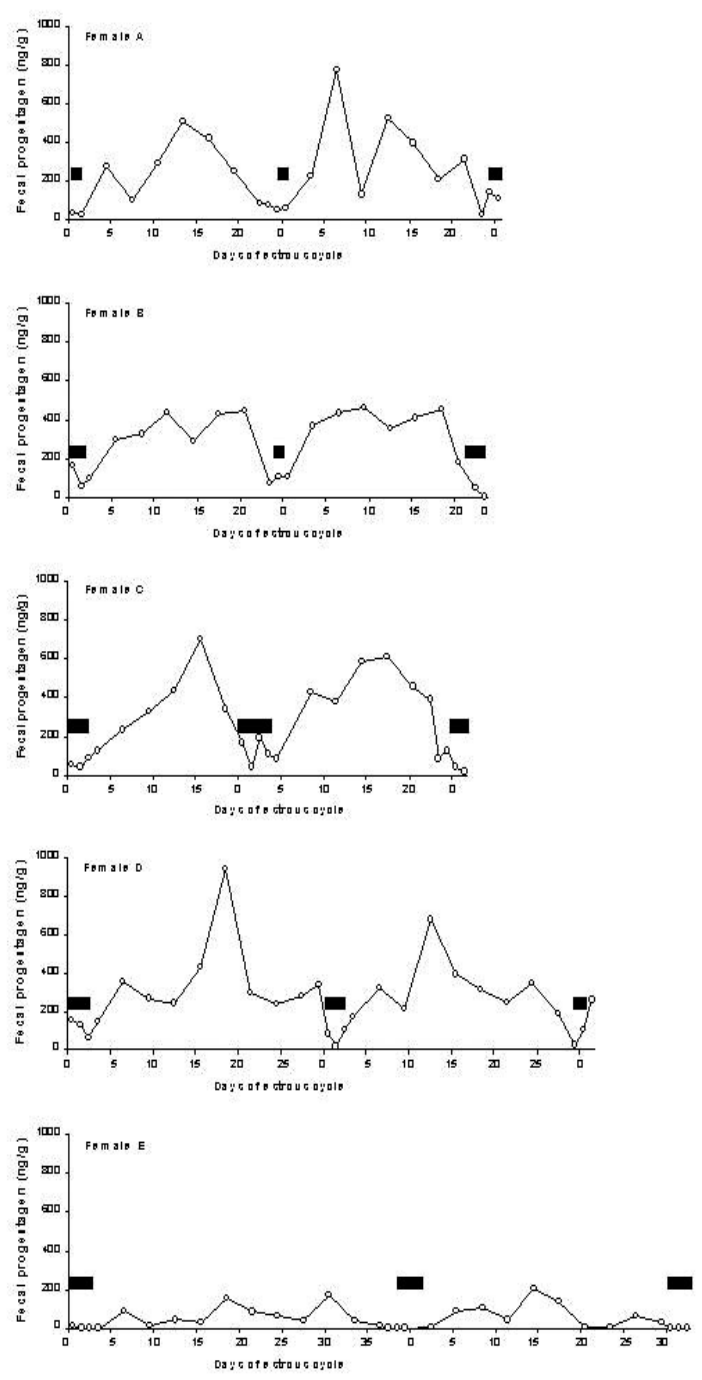

Figura 3 - Perfis individuais das concentrações de progestágenos fecais durante dois ciclos estrais consecutivos em fêmeas de veadocatingueiro (Mazama gouazoubira). Barras horizontais pretas representam os períodos de estro comportamental (fonte: Pereira et al., 2006b). 
felinos selvagens) que eram até pouco tempo totalmente desconhecidas devido aos perigos e limitações vinculados a contenção físico-química estão atualmente disponíveis em literatura (Morais et al., 1996; Morais et al., 2002; Morato et al., 2004; Morato et al., 2004; Moreira 2001). A caracterização de diferentes eventos reprodutivos em espécies brasileiras ameaçadas de estimação tais como gato-do-mato-pequeno (Leopardus tigrinus) e gato-maracajá (Leopardus wiedii) são excelentes exemplos da praticidade dessa metodologia em casos extremos (Figura 4) (Moreira, 2001).

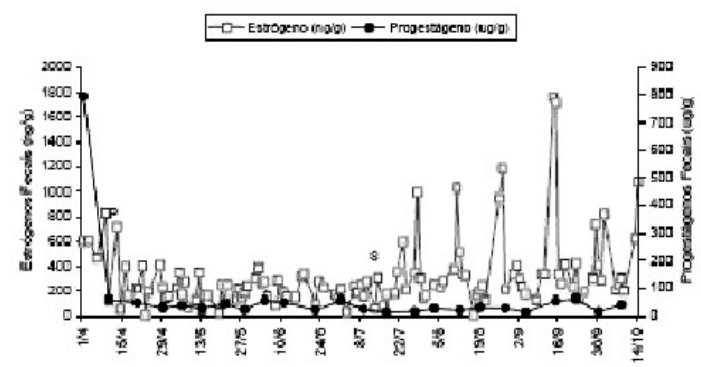

Figura 4 - Perfis da excreção fecal de estrógenos (quadrados vazios) e progestágenos (círculos preenchidos) em fêmeas de gato marcajá (Leopardus wiediii) mantidos em cativeiro. $\mathrm{P}$ - representa o parto / $\mathrm{S}$ - representa a separação dos filhotes (fonte: Moreira, 2001).

\section{Literatura citada}

CHELINI, M.O.M.; SOUZA, N.L.; ROCHA, A.M. et al. Quantification of fecal estradiol and progesterone metabolites in Syrian hamster (Mesocricetus auratus). Brazilian Journal of Medical and Biological Research, v.38, p.1711-1717, 2005.

CHELINI, M.O.M.; SOUZA, N.L.; CORTOPASSI, S.R.G. et al. Assessment of the physiologic stress response by

quantification of fecal corticosteroids. Journal of the American Association for Laboratory Animal Science, v. 45, n.3, p.7-10, 2006.

GUIMARÃES, M.A.B.V.; OLIVEIRA, C.A.; RIBEIRO, E.A.A. et al. Monitoring ovarian function in the capuchin monkey (Cebus apella) using fecal and urinary hormone analyses. In: THE SECOND INTERNATIONAL SYMPOSIUM ON ASSISTED REPRODUCTIVE TECNOLOGY FOR THE CONSERVATION AND GENETIC MANAGEMENT OF WILDLIFE, Omaha, Proceedings... 2002, p. 290.

HODGES, J.K. Determining and manipulating female reproductive parameters. In: KLEIMAN, D.G.; ALLEN, M.E.; THOMPSON, K.V. et al. (Eds) Wild mammals in captivity principles and techniques. The University of Chicago Press, Chicago, United States of America, 1996. p. 418-428.

KORNDORFER, C.M. Avaliação de métodos de extração para a dosagem de progesterona em fezes de coelho (Oryctolagus cuniculus). Dissertação - Centro de Energia Nuclear na Agricultura, Escola Superior de Agricultura Luiz de Queiroz, Piracicaba. 83 p. 1992.
LEE, J.V.; WHALING, C.S.; LASLEY, B.L. et al. Validation of an enzyme immunoassay for measurement of excreted estrogen and testosterone metabolites in the white-tailed sparrow (Zonotrichia leucophrys oriantha). Zoo Biology, v. 14, p.97-106, 1995.

MEIRELLES C.F. Controle reprodutivo em capivras (Hydrochoerus hydrochaeris hydrochaeris Lineu, 1766) atraves da determinação de progesterona nas fezes. Dissertação - Escola Superior de Agricultura Luiz de Queiroz, Piracicaba, 87p.1993.

MILLSPAUGH, J.J.; WASHBURN, B.E. Use of glucocorticoid metabolite measures in conservation biology research: considerations for application and interpretation. General and Comparative Endocrinology, v.138, p.189-199, 2004.

MOSTL, E.; PALME, R. Hormones as indicators of stress. Domestic Animal Endocrinology, v.23, p.67-74, 2002.

MORAIS, R.N.; MOREIRA, N.; MORAES, W. et al. Testicular and ovarian function in South American felids assessed by fecal steroids. In: ANNUAL CONFERENCE OF THE AMERICAN ASSOCIATION OF ZOO VETERINARIANS, Puerto Vallarta, Proceedings, 1996, p. 561-565.

MORAIS, R.N.; MUCCIOLO, R.G.; GOMES, M.F.L. et al. Seasonal analysis of semen characteristics, serum testosterone and fecal androgens in the ocelot (Leopardus pardalis), margay (L. wiedii) and tigrina (Leopardus tigrinus). Theriogenology, v.57, p.2027-2041, 2002.

MORATO, R.G.; BUENO, M.G.; MALMHEISTER, P. et al. Changes in the fecal concentrations of cortisol and androgen metabolites in captive male jaguars (Panthera onca) in response to stress. Brazilian Journal of Medical and Biological Research, v.37, p.1903-1907, 2004.

MOREIRA, N. Reprodução e estresse em fêmeas de felídeos do gênero Leopardus. Tese (Doutorado em Zoologia) Universidade Federal do Parana, Curitiba. 231 p. 2001.

MUHLBAUER, M.; DUARTE, D.P.F.; GILMORE, D.P. et al. Fecal estradiol and progesterone metabolite levels in the threetoed sloth (Bradypus variegatus). Brazilian Journal of Medical and Biological Research, v.39, p.289-295, 2006.

PALME, R. Measuring fecal steroids: guidelines for practical application. Annals New York Academy of Sciences, v. 1046, p.1-6, 2005.

PALME, R. et al. Stress hormones in mammals and birds: comparative aspects regarding metabolism, excretion and noninvasive measurement in fecal samples. Annals New York Academy of Sciences, v.1046, p.24-30, 2005.

PEREIRA, R.J.G.; DUARTE, J.M.B.; NEGRÃO, J.A. Seasonal changes in fecal testosterone concentrations and their relationship to the reproductive behavior, antler cycle and grouping patterns in free-ranging male Pampas deer (Ozotoceros bezoarticus bezoarticus). Theriogenology, v.63, p.2113-2125, 2005.

PEREIRA, R.J.G.; DUARTE, J.M.B.; NEGRÃO J.A. Effects of environmental conditions, human activity, reproduction, antler cycle and grouping on fecal glucocorticoids of freeranging Pampas deer stags (Ozotoceros bezoarticus bezoarticus). Hormones and Behavior, v.49, p.114-122, 2006a.

PEREIRA, R.J.G.; POLEGATO, B.F.; SOUZA, S. et al. Monitoring ovarian cycles and pregnancy in brown brocket deer (Mazama gouazoubira) by measurement of fecal progesterone metabolites. Theriogenology, v. 65, p. 387-399, $2006 b$.

PETER, A.T.; KASPUTIN N.; CRISTER J.K. Analysis of sex steroid metabolites excreted in feces and urine of nondomesticated animals. The Compedium, v.18, n.7, p.781791, 1996.

PINHO, M.P. Efeito da pipotiazina na modulação das respostas ao estresse de veados- catingueiro (Mazama gouazoubira) em cativeiro. Tese (Doutorado em Cirurgia Veterinária) - Faculdade de Ciências Agrárias e Veterinárias,

๑ 2007 Sociedade Brasileira de Zootecnia 
Universidade Estadual Paulista, Jaboticabal. 68f. 2004.

RIBEIRO E.A.A. Uma análise da relação entre o comportamento reprodutivo e os níveis de progestinas fecais em um grupo silvestre do mico-leão-dourado (Leontopithecus rosalia). Tese - Instituto de Biociências, Universidade de São Paulo, USP, 1994.

SCHWARZENBERGER, F.; MÖSTL, E.; PALME, R. et al. Faecal steroid analysis for non-invasive monitoring of reproductive status in farm, wild and zoo animals. Animal Reproduction Science, v.42, p.515-526, 1996.
WASSER, S.K.; HUNT, K.E.; BROWN, J.L. et al. Ageneralized fecal glucocorticoid assay for use in a diverse array of nondomestic mammalian and avian species. General and Comparative Endocrinology, v.120, p. 260-275, 2000.

WASSER, S.K.; HUNT, K.E.; CLARKE, C.M. Assessing stress and population genetics through noninvasive means. In: AGUIRRE, A.A.; OSTFELD, R.S.; TABOR, G.M. et al. (Eds.) Conservation medicine - ecological health in practice. Oxford University Press, New York, United States of America, 2002. p.130-144. 